\title{
Reflection-mode scanning near-field optical microscopy: Influence of sample type, tip shape, and polarization of light
}

\author{
C. Durkan ${ }^{\text {a) }}$ and I. V. Shvets \\ Department of Physics, Trinity College, Dublin 2, Ireland
}

(Received 3 February 1997; accepted for publication 28 October 1997)

\begin{abstract}
A reflection-mode aperture-type scanning near-field optical microscope (R-SNOM) based on the external collection of the reflected light is presented. The light detection is based on an elliptical mirror setup, with the tip and sample at one focus, and a photomultiplier tube at the other. Results are presented on the general imaging properties of this microscope. The results presented concentrate on an analysis of the gap-width dependence of the optical signal, on resolution and on the contrast mechanisms which may be used in R-SNOM imaging, including reflectivity, polarization/magneto-optics, and topographic effects. (C) 1998 American Institute of Physics. [S0021-8979(98)09503-6]
\end{abstract}

\section{INTRODUCTION}

Many advances have been made in the area of scanning near-field optical microscopy (SNOM) over the past decade. ${ }^{1-3}$ The principle of this technique is to scan a subwavelength sized light source above a sample at a height of a few $\mathrm{nm}$. By collecting the light transmitted through the sample or reflected from it, an optical image of the sample may be obtained where the resolution is determined by the source size and the source-sample separation, rather than the wavelength, as is the case in conventional far-field microscopy. Typically, this source consists of an aperture of dimensions less than $150 \mathrm{~nm}$ in a tapered and metal-coated optical fiber. The high divergence of light in the aperture region requires the use of an accurate method of controlling the tip-sample separation (gap width). A technique called shear-force ${ }^{4-6}$ is often used for this purpose. Consequently, it is possible to record the sample surface topography simultaneously with the optical information. The two may then be compared in order to elucidate any purely optical information about the sample. This is an important step, as there is usually a strong correlation between topography and optical images in SNOM. However, the shear-force mechanism is chemically sensitive, ${ }^{7,8}$ hence there may be variations in the tip-sample separation when imaging samples consisting of a mixture of materials. This may result in an artificial contrast in SNOM images due to the strong gap-width dependence of the optical signal, which is analyzed in Sec. II. In this paper, we present results of the study of the imaging mechanisms of R-SNOM, starting with an analysis of the gap-width dependence of the optical signal used for generating images. We then show some effects of the sample type, tip shape, and polarization of the emitted light. It is known that SNOM imaging with a distance regulation mechanism, of samples containing topographic variations leads to artefacts in imaging where the resolution is due mainly to coupling between the optical signal and topography. ${ }^{9,10}$ For this reason, we present results on several different contrast mechanisms. We will return to this point in Sec. III.

\footnotetext{
a) Author to whom correspondence should be addressed; Present address: Engineering Department, University of Cambridge, Cambridge CB2 1PZ, UK.
}

\section{EXPERIMENT}

\section{A. Setup}

The experimental setup is shown in Fig. 1. The fiber tips which are produced with an in-house developed computercontrolled pulling machine ${ }^{11}$ are coated with 50-150 nm of aluminum in such a way as to leave an aperture at the tip apex. It should be mentioned at this point that the thickness, $t$, on a fiber tip is related to the thickness, $d$, as detected on a thickness monitor by $t=(d / \pi) \cos \alpha$, where $\alpha$ is the angle between the normal to the tip axis and the evaporation source.

Distance regulation for maintaining a constant tipsample separation is based on shear force, and the scheme is described elsewhere. ${ }^{7}$ The sensitivity of the detection scheme is typically a few angstroms both laterally and vertically.

Light from a $10 \mathrm{~mW}$ diode laser operating at $635 \mathrm{~nm}$ and electronically modulated at $5 \mathrm{kHz}$ is coupled into the cleaved end of the fiber. The optical signal for R-SNOM is collected with an elliptical mirror which is coaxial with the fiber tip. The tip and sample are located at one focus of the mirror. This enables the collection of the reflected light from a complete solid angle of $2 \pi / 3$. The collection symmetry helps to reduce shadowing effects, which are of paramount importance in R-SNOM. This arrangement has been found to be superior to the situation where a single objective collects the reflected light. ${ }^{12}$ The collected light is then detected with a photomultiplier tube (PMT) located at the second focus of the mirror. Lock-in detection is then used to enhance the signal-to-noise ratio (SNR) of the detected signal. With this scheme, a SNR of a few hundred is readily achieved.

\section{B. Gap-width dependence of the optical signal}

As (R-)SNOM images are generated by measuring the detected optical signal at each image point, it is essential to have an understanding of the signal characteristics, particularly with respect to tip-sample distance, which we refer to as the gap width. For R-SNOM, the typical detected optical signal level is $0.1-1 \mathrm{nW}$, for $1-10 \mathrm{~mW}$ of power coupled into the fiber. This corresponds to a transmission through the tips and subsequent detection efficiency of the order $10^{-6}$. On monitoring the signal due to the light transmitted through 


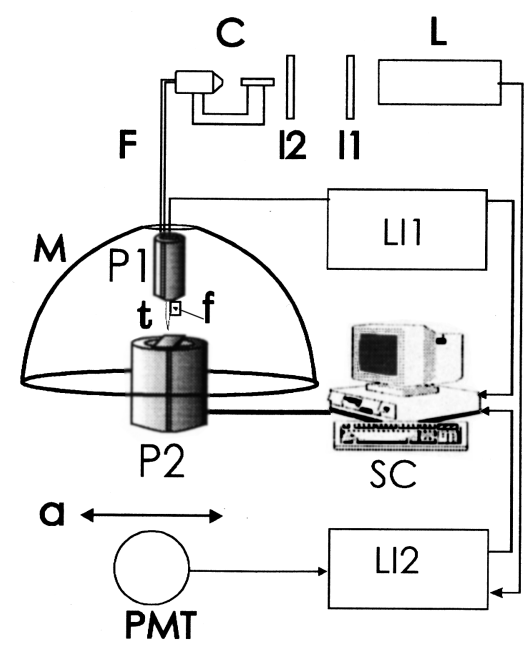

FIG. 1. Schematic of the experimental setup. P1: shear-force dither piezo, P2: sample scanner piezo, t: fiber tip, f: shear-force detector, F: optical fiber, C: laser coupler, L: laser, L11, L12: lock-in amplifiers, M: elliptical mirror, a: polarization analyzer, $\lambda / 2$ and $\lambda / 4$ : wave plates, PMT: photomultiplier tube, SC: scan control unit and data acquisition.

the tip and subsequently reflected from the sample while the tip is being approached to the sample, interference oscillations are clearly seen, as shown in Fig. 2(a). Here, the period is about $\lambda / 2$ with the first maximum at a gap width of about $\lambda / 4$. The slight change in spacing between maxima shown here is primarily due to nonlinearity of the PZT piezotube. These oscillations are due to interference between light emitted from the tip and light reflected from the sample. Two points are most important for the analysis of such a curve: the position of the first maximum and the period of the oscillations.

These two characteristics depend primarily on the following factors: (1) the distance of the optical aperture from the sample at shear-force contact (typically 5-10 $\mathrm{nm}$ from
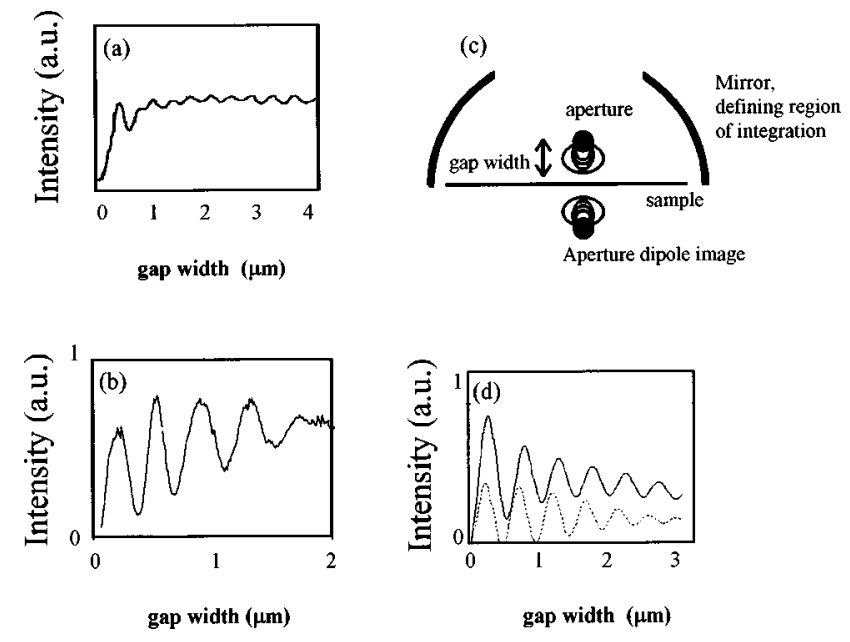

FIG. 2. Optical approach curves in reflection: (a) measured with mirror summing over all reflected angles from $30^{\circ}$ to $90^{\circ}$; (b) measured at $30^{\circ}$ to tip axis with objective of $\mathrm{NA}=0.2$; (c) illustration of tip and tip image used in calculation (d) calculated signal for sum over $30^{\circ}-90^{\circ}$ (solid line) and at $30^{\circ}$ (dotted line, scale expanded by 5 for clarity). actual contact). This depends on the length of protrusions, generally grains in the metal coating at the tip apex which act as shear-force sensors, as these protrusions come into the point of shear-force contact when the optical aperture is still some distance away from the surface and (2) the angular dependence of the light emitted from the tip. It has already been demonstrated that the far-field emission pattern of a SNOM tip depends on the aperture size. ${ }^{13}$ A large aperture will have a narrower angular emission pattern than a small one. This can be readily understood from Fourier optics. ${ }^{14} \mathrm{~A}$ small aperture contains higher spatial frequencies, and hence a broader angular spectrum than a large one. Hence, the angular range of emission will be different for different tips, and a consequence of this is that different tips will exhibit different characteristics in the gap-width dependence of optical approach curves. A detector oriented at a given angle, $\theta$ to the tip axis will observe a maxima spacing of $(\lambda / 2 \cos \theta)$ for the case of one reflection from the sample. We have observed the position of the first maximum in the range of gap width from $\lambda / 4$ to $3 \lambda / 4$, and the spacing between maxima in the range $\lambda / 1.6-\lambda / 2$.

The detected signal is a superposition between light emitted at all angles to the tip axis. By considering this signal as the integral of the intensity due to the interference between coherent point sources of light, i.e., two orthogonal dipoles in the aperture plane ${ }^{13}$ and their image in the sample [Fig. 2(c)], as a function of the detection solid angle for different gap widths, then the overall detected signal still shows interference behavior. This is illustrated in Fig. 2(c), which shows the sum of the calculated signal, summed over $\theta=30^{\circ}-90^{\circ}\left(90^{\circ}\right.$ corresponding to the plane of the sample), and for all azimuths corresponding approximately to the measurable angle in our setup, and for the gap width varying from 0 to $3.3 \mu \mathrm{m}$. Also shown is the calculated signal at $30^{\circ}$. This is assuming that all points in the calculation are equidistant from the tip-sample junction. Figure 2(b) shows a typical reflection approach curve measured with an objective of NA $=0.2$ at $30^{\circ}$ to the tip-sample axis. There is reasonable qualitative agreement with the measured curve. One result of this simple model is that as the aperture size decreases and its angular spread increases, the period of the observed maxima should decrease. This fits well with our observations.

The optical signal at shear-force contact is generally $20 \%-30 \%$ of the value at a gap width of a few microns. Between gap widths from contact to about $\lambda / 4$ there is a steep increase in the optical signal which is only partially explained by the model presented earlier. Shadowing of the reflected light by the tip itself necessarily plays a major role. Simultaneous observation of the transmitted light shows no such decrease, and the signal consists of a simple interference pattern, of period $\lambda / 2$. From the analysis of such optical approach curves as shown here, it can be deduced that at shear-force contact, if the tip-sample separation varies by as little as $1 \mathrm{~nm}$, then the detected optical signal (intensity) can vary by up to a few percent. The exact value depends on the tip shape and the aperture size. For many samples of practical interest this is of at least the same order as the optical contrast in the sample. Hence, it is essential to be able to 

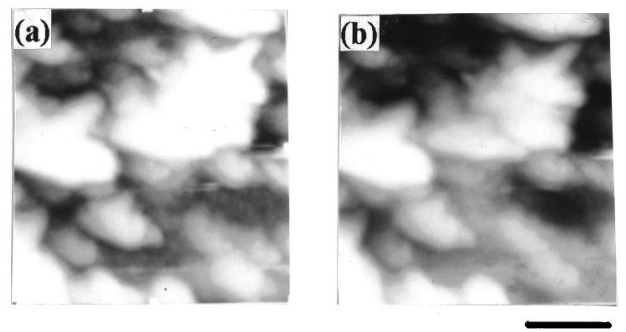

$500 \mathrm{~nm}$

FIG. 3. (a) and (b) shear force and R-SNOM images of aluminum film. Scan size $=1.6 \mu \mathrm{m} \times 1.6 \mu \mathrm{m}$.

maintain the tip-sample separation constant to greater than a few angstroms. We shall return to this point in Sec. III B. It should be noted at this point that the corresponding change in the transmitted optical signal due to gap-width variations is almost an order of magnitude less than for reflection.

From considering the aperture as a combination of radiating dipoles, and the detected optical signal as a superposition between this and image dipoles in the sample, it may be seen that the majority of light is reflected in a lobe along the tip axis once the gap width decreases below $\lambda / 2$. The presence of the tip blocks this light from reaching the detector, and hence the detected optical signal for this arrangement is at least an order of magnitude less than for transmissionmode SNOM.

\section{IMAGING WITH R-SNOM}

In this section, we deal with the results of imaging different types of samples with the microscope. We initially show that R-SNOM is highly sensitive to the sample topography. We then proceed to demonstrate reflectivity and polarization contrast. We finally demonstrate that it is possible to obtain high-resolution images of samples containing optical contrast without any associated topography.

\section{A. Topographic samples}

Figure 3 shows shear force and R-SNOM images of an aluminum film, sputtered onto a glass substrate, and of mean roughness $60-100 \mathrm{~nm}$. Here, there is a 1:1 correspondence between the optical and shear-force images. Features as small as $40 \mathrm{~nm}$ are clearly resolved in the R-SNOM image. The origin of this "optical contrast" has three sources: (1) the shear-force sensor and the optical aperture are generally not at exactly the same location on the tip: the shear-force sensor is typically a protrusion in the metal coating forming the rim surrounding the aperture. Hence, as the tip scans across a nonflat sample, while the shear-force control maintains a constant separation between the tip and sample, the distance from the aperture to the sample will not be constant. Also, while scanning across samples containing significant topography, the point on the tip acting as a shear-force sensor will shift. With a $3 \% / \mathrm{nm}$ variation in optical intensity, it is possible to get a large contrast, and (2) the tip shadows a large portion of the reflected light. As the tip scans across the sample, protrusions will help scatter light away from the tip, and hence appear bright, whereas depressions "focus" light

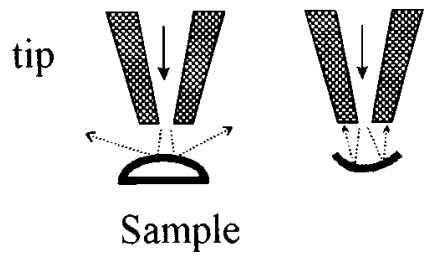

FIG. 4. Illustration of effect of topography on light emitted from aperture.

onto the end of the tip, increasing the shadowing and hence depressions appear dark. This is illustrated in Fig. 4. This is a gross simplification as ray optics do not hold on to such a small scale, but this is nonetheless an intuitive way of explaining the results and is consistent with our observations on numerous samples, (3) there will be variations in reflectivity across the sample, due to the different angles of incidence. This is a pure optical contrast and will also contribute to the image. However, on that basis, it should be expected that the brightest parts of the image should correspond to the sides of topographic hills and valleys, rather than the top as is observed here. The primary origin of the imaging mechanism therefore is point 1, as illustrated in Fig. 5. Light is not completely confined in the vicinity of the SNOM aperture. There are propagating waves transmitted through the aperture which are not confined below $\lambda / 2$. The overall confinement of energy in the aperture vicinity is due to a sum of propagating and nonpropagating waves. As a protrusion on the tip follows the surface topography faithfully, even though the aperture to sample separation may remain constant (which is not very likely) the mean distance between the diffraction-limited propagating waves and the sample varies. Hence, the SNOM is acting as an interferometer and the resolution is determined more by the topography than the aperture size. An obvious way to overcome this problem is to scan at constant height. ${ }^{9,10}$ Figure 6 shows two SNOM images of a carbon test grating of a period of $460 \mathrm{~nm}$, groove width $80-100 \mathrm{~nm}$, and groove depth of 40-80 nm. Figure 6(a) was obtained using shear-force feedback, and Fig. 6(b) without any feedback, at a gap width close to actual contact. These images were taken using two different tips, hence the difference in contrast. It can be seen that the resolution of Fig. 6(b) is below $100 \mathrm{~nm}$. The rest of the results presented here were obtained using shear-force distance regulation.
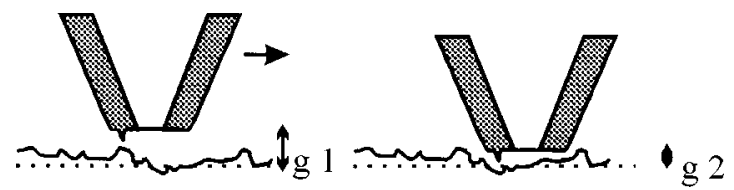

FIG. 5. Illustration of origin of topographic artefacts. The dotted line shows the average sample surface as seen by the diffraction limited waves from the aperture. As the tip follows surface topography, the gap width varies from $\mathrm{g} 1$ to g2, and this combined with the distance sensitivity from Fig. 2(a) produces a sizable topographic coupling. 


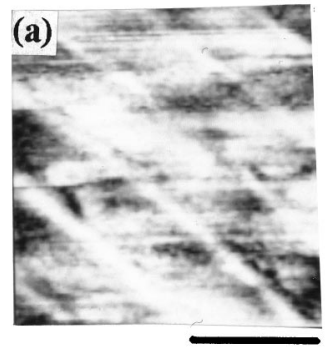

$1 \mu \mathrm{m}$

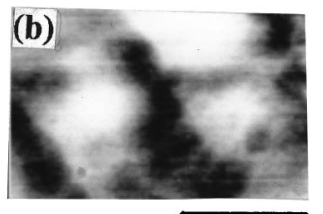

$500 \mathrm{~nm}$
FIG. 6. SNOM images of gratings (a) with and (b) without shear-force feedback, almost at actual contact. Scan sizes are for (a) $2 \mu \mathrm{m} \times 2 \mu \mathrm{m}$ and (b) $1 \mu \mathrm{m} \times 0.6 \mu \mathrm{m}$.

\section{B. Samples containing topography and optical contrast}

\section{Reflectivity contrast}

Figure 7 shows shear force and R-SNOM images of an $e$-beam etched chromium test pattern of height $80 \mathrm{~nm}$ fabricated on a glass substrate. ${ }^{15}$ For both images, the minimum edge sharpness is of the order $15 \mathrm{~nm}$. This would imply an aperture diameter of approximately $5-10 \mathrm{~nm}$ (given a skin depth of approximately $6 \mathrm{~nm}$ for aluminum). It is highly improbable to obtain such a small aperture size, and it is interesting to note that the resolution apparent in both Figs. 7 (a) and 7(b) is identical, which would mean that the force sensor is exactly the same size as the optical aperture. The observed SNOM resolution is mainly due to a topographic coupling with the optical signal and is related to the distance regulation mechanism. As the probe tip scans across the grating, when it is above the glass between two $\mathrm{Cr}$ lines, the backreflected light is partially prevented from reaching the detector due to shadowing by the tip itself and the $\mathrm{Cr}$ sidewalls. This is not the case when the tip is above a $\mathrm{Cr}$ line, as the shadowing is purely due to the tip. Essentially, the sample and tip combination prevent light from reaching the detector when the sample contains significant topography variations which the tip follows. Thus, effectively, the resolution is enhanced by the strong dependence of the optical signal upon the surface topography through shadowing. It is therefore difficult to know how much contrast to expect for the structure.
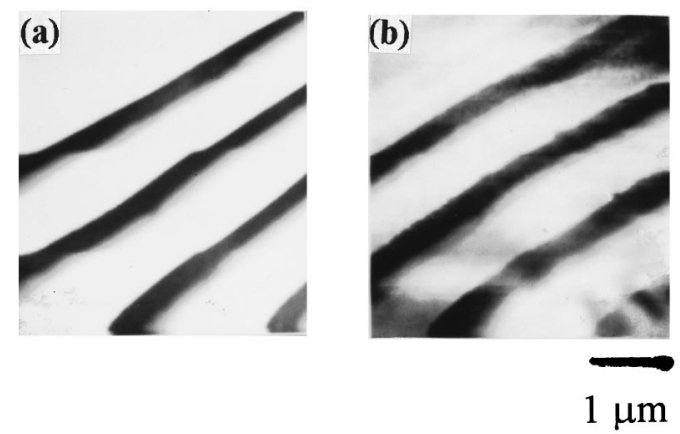

FIG. 7. (a) and (b) shear force and R-SNOM images of $\mathrm{Cr}$ grating on glass. Scan size $=4 \mu \mathrm{m} \times 4 \mu \mathrm{m}$.
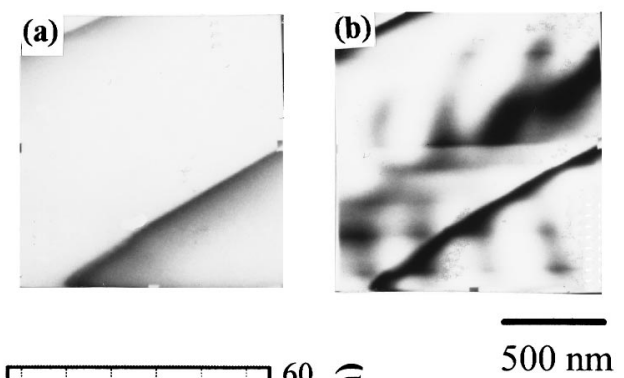

(c)

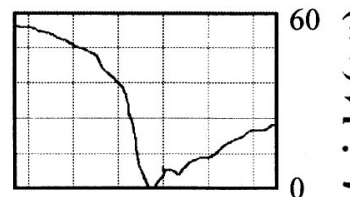

(d)

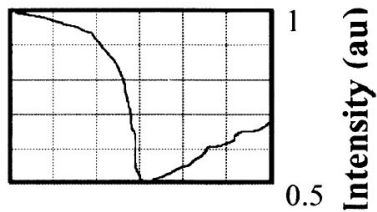

$0 \quad 100 \quad 200$

\section{Distance along line (nm)}

FIG. 8. (a) and (b) shear force and R-SNOM images of Cr grating on glass with asymmetric tip. Scan size $=1.5 \mu \mathrm{m} \times 1.5 \mu \mathrm{m}$ (c) and (d) line profiles across sharply resolved edge in both images.

The tip shape plays a major role in image formation. This is already indicated by Fig. 7, as both edges of each $\mathrm{Cr}$ line are clearly different for both images. For one edge of each line, the measured edge sharpness is about $15 \mathrm{~nm}$, whereas the other edge has a sharpness of about $70 \mathrm{~nm}$. This effect is further illustrated in Fig. 8. This shows shear force and SNOM images of a similar area of the sample as shown in Fig. 7, but with a different tip. The scanning parameters were identical to those for Fig. 7. The tip in this case is so asymmetric that it can now only image one edge of each grating line. At the same time, light leaking from the tip shaft gives rise to the interference pattern along the lines.

\section{Polarization contrast}

The results of the previous sections show that topographic effects often override true optical contrast, introducing imaging artefacts. For this reason, we now present results of a study of polarization-dependent imaging where we show that for this case, optical effects can be stronger than topographic artefacts.

We have shown elsewhere ${ }^{16}$ that it is possible to distinguish between light emitted from a SNOM aperture and light leaked from the tip shaft, when imaging metal samples due to the change in polarization of light emitted from the aperture on reflection from a metal surface. This change can be rather large (up to $90^{\circ}$ ) once the tip-sample separation decreases to a few $\mathrm{nm}$. In this section we demonstrate polarization dependent imaging of a test grating.

It has been shown theoretically that the emission pattern of light from a SNOM aperture depends on its proximity to structures, the effect being much larger for metals than dielectrics; and on the polarization state of the light emitted from the tip. ${ }^{17-19}$ The origin of these effects is first the matching of electromagnetic boundary conditions at the 

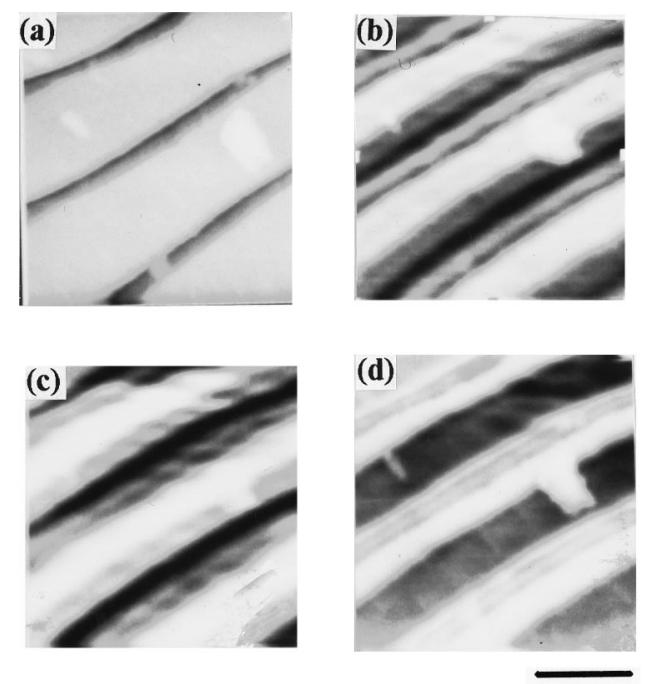

$1 \mu \mathrm{m}$

FIG. 9. (a) Shear force and (b), (c) and (d) R-SNOM images of Cr grating on glass for polarization of light at (b) $45^{\circ}$, (c) $10^{\circ}$, and (d) $70^{\circ}$ to lines. All scan sizes $=3 \mu \mathrm{m} \times 3 \mu \mathrm{m}$.

metal/dielectric-air interface and second, the modification of the angular spectrum of the aperture due to the varying optical properties of the sample surface.

The sample used for these experiments was a similar region of the $e$-beam etched sample as shown in Fig. 7. Here, the width of the metal lines is $1 \mu \mathrm{m}$ and the gap between lines is $250 \mathrm{~nm}$. Figure 9 shows shear force and R-SNOM images of this sample. In Figs. 9(b), 9(c), and 9(d), the light emitted from the tip was polarized at $45^{\circ}, 10^{\circ}$, and $70^{\circ}$ to the lines, respectively. No analyzer was used for these experiments. There is excellent qualitative agreement between the results here and simulations of similar systems. ${ }^{17}$ For the case of light polarized parallel to the metal lines, the contrast is high, the resolution is low, and the optical image bears a relatively close resemblance to the object. For the case of the light polarized perpendicular to the lines, the contrast is lower, the resolution is higher, and there is no longer an obvious resemblance of the optical image to the object. Here, the glass between the metal lines is even brighter than the metal lines themselves. These results may be understood by remembering that the far-field emission characteristics of an aperture may be modeled as being due to two dipoles in the plane of the aperture. One electric dipole is along the plane of polarization, and the other, a magnetic dipole of half the strength is along the orthogonal direction. As the tip scans above the glass, between two metal lines, each source dipole in the tip induces image dipoles in the metal strips on either side, as well as weaker ones in the glass below. Any source dipole oriented along the metal lines will induce image dipoles of opposite orientation in the metal, whereas a source dipole perpendicular to the metal induces a dipole of the same orientation. Hence, with the polarization along the lines, the source dipoles are essentially canceled by the effect of their induced dipoles on either side. With the polarization perpendicular to the lines, the source and it's images con- structively interfere. The magnitude of the change in signal expected when the polarization switches from one direction to the other depends on the size of the induced dipoles, i.e., on the polarizability of the metal. The experimentally measured intensity increased by almost $50 \%$ when the tip was maintained between the lines and the polarization was rotated from parallel to perpendicular to the lines. A negligible change was observed for the case of the tip maintained above the center of one of the metal lines while the polarization was rotated. Even dust particles on the glass are now visible, while they were not for the previous case. The bright rims along the metal edges are simply due to the fact that the shadowing by the tip is less efficient when the tip is above a metal line than between two of them. They prove that the emission characteristics of SNOM tips are affected over a long range (here of the order $500 \mathrm{~nm}$ ). The asymmetry in the rim dimensions is again due to an asymmetric tip shape.

It is interesting to note that the "resolution" of these images depends on the polarization state of light. This means that the polarization effects are larger than, or at least comparable to topographic effects, and that the resolution can be "tuned" by varying the polarization state of the emitted light. This may be ruled out as being due to the different degrees of confinement of light along and perpendicular to the polarization direction in the vicinity of the aperture, as the energy is less strongly confined in the direction of polarization. Hence, the concept of resolution in SNOM is rather complicated, as it depends on the aperture size, the tip geometry, the surface topography, the polarization state of light, whether or not distance regulation is used, the tipsample separation, and the tip-sample tilt. Therefore, in order to make any meaningful claims about resolution, samples without any topography should be investigated.

\section{Sample containing purely optical contrast}

As was explained in previous sections, surface topography without associated strong optical contrast complicates the issue of resolution in R-SNOM. Several systems contain optical contrast without any associated topography. One of these is single-molecule detection, which has previously been demonstrated with SNOM ${ }^{21}$ another is magneto-optics. Faraday effect SNOM imaging has already been demonstrated, ${ }^{22}$ but little work has been done to date on Kerr effect $\mathrm{SNOM}^{23,24}$ which is potentially more useful as most conventional magneto-optic materials are relatively opaque. Here we show that Kerr effect SNOM imaging is indeed possible on flat samples, and with a resolution of $60 \mathrm{~nm}$ or more. The sample used for these experiments was a magnetic $\mathrm{Co} / \mathrm{Pt}$ multilayer ${ }^{25}$ with a series of tracks of prewritten bits of dimension and spacing in the range $400-800 \mathrm{~nm}$, depending on the particular area of the sample. This sample is magnetized out of plane, and the polar Kerr rotation for normally incident light at $635 \mathrm{~nm}$ is $\sim 0.25^{\circ}$. It is known that SNOM fiber tips may reach temperatures of over $500 \mathrm{~K}^{26}$ due to the light within the tip being absorbed by the metal coating. As the Curie point of our sample was $650 \mathrm{~K}$, there was the possibility of unintentionally erasing the bit pattern while trying to image it. To avoid this, we coupled the minimum comfortable amount of light into the tip, resulting in a detected signal of $1.5 \mathrm{nW}$. The aperture diameter was of the 

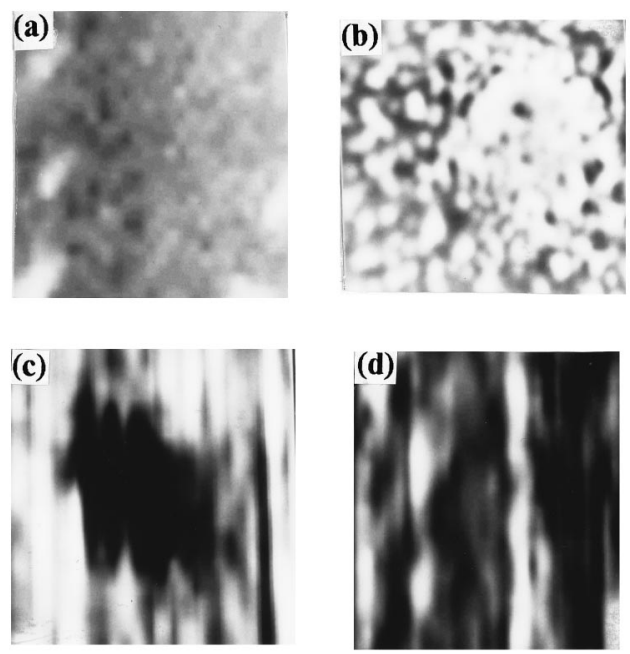

FIG. 10. (a) Shear force, (b) MFM, (c) and (d) R-SNOM images of magnetic bit in Co/Pt multilayer. Scan size $=700 \times 700 \mathrm{~nm}$ for (a), (c) and (d), and $1.6 \mu \mathrm{m} \times 1.6 \mu \mathrm{m}$ for (b). Analyzer rotated by $90^{\circ}$ between (c) and (d).

order 50-60 nm. For this signal level, with lock-in detection at a time constant of $10 \mathrm{mS}$, we had an optical signal-tonoise ratio of 200 . This means that a rotation of the polarization axis of $0.25^{\circ}$ results in a change in the detected optical signal which is of the same value as the noise level, therefore, this is just on the limit of detection. For many magneto-optic recording media, this is just enough to detect a rotation in the polarization state of the reflected light.

The thickness of the metal coating at the tip apex was of the order $60-80 \mathrm{~nm}$, the minimum amount possible for suitable light confinement, while also minimizing the depolarization of the reflected light. Figure 10 shows MFM (magnetic force microscope), shear force and R-SNOM images of a bit on this sample. With shear force, the gap width was regulated at approximately $5 \mathrm{~nm}$. The MFM image is of a bit of diameter $\sim 600 \mathrm{~nm}$, and the R-SNOM images are of a bit of $\sim 400 \mathrm{~nm}$ diameter. The MFM image shows the intrinsic domain structure of the multilayer, where the characteristic dimension of the domains is $\sim 60 \mathrm{~nm}$. The shear-force image shows that there is no topography associated with the bit, and the R-SNOM images are taken for two orthogonal states of the analyzer, to demonstrate that this is indeed a magnetic bit. Larger scan areas enabled imaging of the track pattern, and the spacing between bits, their dimension and the orientation of the tracks was consistent with that observed with conventional Kerr microscopy. In the R-SNOM image Fig. 10(c), the domain structure can also be inferred from the roughness of the bit (the pixel size is $10 \mathrm{~nm}$ ). From the optical edge sharpness of the bit, the resolution can be estimated at greater than $60 \mathrm{~nm}$. This is a significant step, as to date the only methods for imaging bits/domains on a sub-250 $\mathrm{nm}$ scale are electron microscopy either with polarization analysis or the Bitter technique, and MFM. R-SNOM offers a complementary technique for the imaging of magnetic domains. This has important consequences for the future, as read heads are approaching the single-domain size, and being magnetically soft, characterization by MFM is not yet routinely feasible.

\section{CONCLUSIONS}

We have presented results on the general imaging properties of reflection-mode SNOM with external collection of the reflected light. We have shown that an understanding of the optical signal used for generating images is important for interpreting images of topographic samples. We have demonstrated that several of the contrast mechanisms used in conventional optical microscopy are possible in R-SNOM. There is a strong coupling between topography and the optical signal. We have also shown that R-SNOM images depend on the tip shape and the polarization state of light. Finally, we demonstrated that it is possible to obtain high resolution images of magnetic bits on a topographically flat magneto-optic sample.

\section{ACKNOWLEDGMENTS}

Financial support from DGXII of the Commission of the European Union, Contract ERBCHRX CT 93-0375 is gratefully acknowledged. The authors also wish to thank B. Hecht for many useful and stimulating discussions on the nature of this work.

${ }^{1}$ D. W. Pohl, W. Denk, and U. Dürig, Proc. SPIE 565, 56 (1985).

${ }^{2}$ A. Lewis et al., Biophys. J. 41, 405a (1983).

${ }^{3}$ E. Betzig and J. K. Trautman, Science 257, 189 (1992).

${ }^{4}$ E. Betzig, P. L. Finn, and J. S. Weiner, Appl. Phys. Lett. 60, 2484 (1992).

${ }^{5}$ R. Toledo-Crow, P. C. Yang, Y. Chen, and M. Vaez-Iravani, Appl. Phys. Lett. 60, 2957 (1992).

${ }^{6}$ C. Durkan and I. V. Shvets, J. Appl. Phys. 79, 1219 (1996).

${ }^{7}$ C. Durkan and I. V. Shvets, J. Appl. Phys. 80, 5659 (1996).

${ }^{8}$ A. Jalocha, M. H. P. Moers, A. G. T. Ruiter, and N. F. van Hulst, Ultramicroscopy 61, 221 (1996).

${ }^{9}$ B. Hecht, D. W. Pohl, H. Bielefeldt, L. Novotny, and Y. Inuoye, J. Appl. Phys. 81, 2492 (1997).

${ }^{10}$ V. Sandoghdar, S. Wegscheider, G. Krausch, and J. Mlynek, J. Appl. Phys. 81, 2499 (1997).

${ }^{11}$ I. V. Shvets and S. Madsen, Irish Patent No. S63296 (1995).

${ }^{12}$ C. Durkan and I. V. Shvets, in Photons and Local Probes, edited by O. Marti and R. Möller NATO ASI Ser. E: Appl. Sci. (Kluwer, Dordrecht, 1995), Vol. 300, pp. 145-150.

${ }^{13}$ C. Obermuller and K. Karrai, Appl. Phys. Lett. 67, 3408 (1995).

${ }^{14} \mathrm{~J}$. W. Goodman, Introduction to Fourier Optics (McGraw-Hill, London, 1968).

${ }^{15}$ Test pattern kindly supplied by I. V. Rangelow and P. Hudek, University of Kassel, Germany.

${ }^{16}$ C. Durkan and I. V. Shvets, J. Appl. Phys. (in press).

${ }^{17}$ L. Novotny, D. W. Pohl, and P. Regli, J. Opt. Soc. Am. A 11, 1768 (1994)

${ }^{18}$ C. Girard, A. Dereux, and O. J. F. Martin, in Photons and Local Probes, edited by O. Marti and R. Möller (Kluwer, Dordrecht, 1995), Vol. 300, p. 1 .

${ }^{19}$ D. Barchiesi and D. van Labeke, in Near-Field Optics, edited by D. W. Pohl and D. Courjon (Kluwer, Dordrecht, 1993), Vol. 242, p. 179.

${ }^{20}$ See, for instance, P. Lorrain, D. P. Corson, and F. Lorrain, Electromagnetic Fields and Waves (Freeman, San Francisco, 1988).

${ }^{21}$ E. Betzig and J. Chichester, Science 262, 1422 (1993).

${ }^{22}$ E. Betzig, J. K. Trautman, J. S. Weiner, T. D. Harris, and R. Wolfe, Appl. Opt. 31, 4563 (1992).

${ }^{23}$ T. J. Silva, S. Schultz, and D. Weller, Appl. Phys. Lett. 65, 658 (1994)

${ }^{24}$ C. Durkan and I. V. Shvets, Appl. Phys. Lett. 70, 1323 (1997).

${ }^{25}$ Sample kindly provided by C. Lodder, MESA Research Institute, University of Twente, the Netherlands.

${ }^{26}$ D. I. Kavaldjiev, R. Toledo-Crow, and M. Vaez-Iravani, Appl. Phys. Lett. 67, 2771 (1995). 